\title{
Measuring socioeconomic inequality in health, health care and health financing by means of rank-dependent indices: A recipe for good practice
}

\author{
Guido Erreygers ${ }^{\mathrm{a},{ }^{*}}$ and Tom Van Ourti ${ }^{\mathrm{b}}$ \\ Tom Van Ourti: vanourti@ese.eur.nl \\ aDepartment of Economics, University of Antwerp, City Campus, Prinsstraat 13, 2000 Antwerpen, \\ Belgium \\ bErasmus School of Economics, Erasmus University Rotterdam, PB 1738, 3000 DR Rotterdam, \\ The Netherlands; Tinbergen Institute, and NETSPAR
}

\begin{abstract}
The tools to be used and other choices to be made when measuring socioeconomic inequalities with rank-dependent inequality indices have recently been debated in this journal. This paper adds to this debate by stressing the importance of the measurement scale, by providing formal proofs of several issues in the debate, and by lifting the curtain on the confusing debate between adherents of absolute versus relative health differences. We end this paper with a 'matrix' that provides guidelines on the usefulness of several rank-dependent inequality indices under varying circumstances.
\end{abstract}

\section{Keywords}

Health inequality; Socioeconomic inequality; Concentration Index

\section{Introduction}

The Concentration Curve and the related Concentration Index (CI) have nowadays attained the status of "workhorse in most health economic studies" (Fleurbaey and Schokkaert, 2009: 73). A characteristic feature of the $\mathrm{CI}$ is that it measures the socioeconomic inequality of health by taking into account every individual's level of health and every individual's rank in the socioeconomic domain. Anyone familiar with the income inequality literature (see e.g., Kakwani, 1980 and Lambert, 2001) will recognize a strong similarity between the CI and the Gini coefficient. In general it can be said that research on income inequality has inspired much of the research on the $\mathrm{CI}$, including the recent work on its welfare economics foundations (Bommier and Stecklov, 2002; Bleichrodt and van Doorslaer, 2006). In this paper we focus on an issue which has received less attention in the literature: the properties of the variables measuring health and socioeconomic position. The matter appears to be of little importance for the socioeconomic dimension, since an ordinal variable suffices to rank individuals according to their socioeconomic position (Wagstaff and Watanabe, 2003; Lindelöw, 2006; O’Donnell et al., 2008: chapter 6). For the health variable, by contrast, the

\footnotetext{
*Corresponding author. Tel: +32-3-265 40 52; fax: +32-3-265 45 85., guido.erreygers@ua.ac.be (Guido Erreygers).

${ }^{17}$ Note that this also implies that we reject the relevance of the 'fixed-range' argument upon which the Wagstaff Index is based. Wagstaff (2005) constructed his index by dividing the standard CI by its mean-dependent upper bound; this procedure yields an index of which the lower and upper bounds are mean-independent and always equal to -1 and +1 .
} 
issue is far less innocuous (Clarke et al., 2002; Wagstaff, 2005, 2009; Erreygers, 2009a, 2009b; van Doorslaer and Van Ourti, forthcoming).

Wagstaff (2005) has shown that the minimum and maximum values of the CI calculated on the basis of a binary variable - say suffering from a chronic illness or not - depend upon the mean of this variable. This holds more generally for any bounded variable (Erreygers, 2009a), and thus complicates the comparison of the values of the CI for populations with different mean health levels. The bounded character of the health variable moreover raises the question of the relation between health and ill health inequality. Erreygers (2009a) has argued that an index of socioeconomic inequality should reveal the same 'magnitude of inequality' when calculated on the basis of the health variable and when calculated on the basis of the associated ill health variable, since health and ill health are just 'mirrors' of one another. The CI does not have this mirror property. Invariance to measurement scale is another desirable requirement put forward by Erreygers (2009a). While the CI and the related Gini index have been developed in the field of income inequality measurement where ratio-scale properties can be taken for granted - multiplying all incomes by a positive number does not affect the value of the Gini index -, health indicators often do not have ratio-scale properties. Van Doorslaer and Jones (2003) and Erreygers (2009a) have shown that the CI is invariant to proportional changes; but not to positive linear transformations that would be needed for cardinal health variables such as the Health Utility Index (Feeny et al. 2002, Furlong et al. 2001). Whereas the standard CI fails the mirror and cardinal invariance tests, both the 'corrected' CI proposed by Erreygers (2009a) and the generalized version of the modified CI proposed by Wagstaff (2005) pass the two tests. Additional value judgements, recently discussed in this journal by Wagstaff (2009) and Erreygers (2009a, 2009b), are needed to discriminate between the two.

The purpose of this paper is threefold. First, we focus on the importance of accounting for measurement scale, and develop guidelines that discriminate between various rankdependent inequality indices based on the measurement scale and the properties of the underlying health variable (and by extension health care and expenditure indicators). Second, we discuss and prove several of the properties of rank-dependent indices for bounded variables that were presented in Erreygers (2009a, 2009b), and provide - if possible - the corresponding properties for unbounded variables. Third, we revisit the discussion of Erreygers and Wagstaff and show its relation with the long-lasting debate in epidemiology (and health economics) on measuring absolute versus relative health differences (among others Wagstaff et al., 1991; Mackenbach and Kunst, 1997; Oliver et al., 2002; Regidor, 2004; Avendano Pabon, 2006; Harper and Lynch, 2007; Mackenbach et al., 2008; Regidor et al., 2009; Harper et al., 2010). We show that a lot of confusion derives from defining absolute and relative inequality on the raw indicators. In order to clarify things, (i) we make the case for transforming health indicators into a 'standardized representation' before defining absolute and relative inequalities, and (ii) we stress the impossibility of measuring relative inequalities only while accepting at the same time that health inequality should be the mirror image of ill health inequality. ${ }^{1}$

The remainder of this paper is organised as follows. In the next section, we focus on the measurement scales of health variables. The third section introduces the class of rankdependent inequality indices, and section 4 discusses the properties and conditions needed to narrow down this class of indices to specific indices. The fifth section discusses the implications of these properties and the measurement scale of health variables for the usefulness of the specific rank-dependent inequality indices. The final section concludes.

\footnotetext{
${ }^{1}$ In this, as in much of the paper, we are following the path indicated by Erreygers (2009a, 2009b).
} 


\section{Properties and measurement scale of variables}

We consider a given population of $n$ individuals and assign to each individual $i=1,2, \ldots, n$ a rank $\lambda_{i}$ based upon this person's socioeconomic position, with the least well-off individual ranked first and the best well-off ranked last. The health level of individual $i$ is represented by the health variable $h_{i}$, a real number. The vector $h=\left(h_{1}, h_{2}, \ldots, h_{n}\right)$ represents the health situation of the whole population. We assume that a higher value of $h_{i}$ indicates a better health situation of individual $i$. The average health of the population is denoted as $\mu_{h}$ and is assumed to be finite.

Health variables can be measured on different scales (Roberts, 1979), i.e. the level of measurement can be:

- nominal, implying that one can classify individuals without being able to order;

- ordinal, allowing to order individuals, but with the differences between individuals being meaningless;

- cardinal, meaning that differences between individuals make sense, but ratios not, such that the zero point is fixed arbitrarily;

- ratio-scale, involving that ratios between individuals have meaning and the zero point corresponding to a situation of complete absence, such that the measurement scale is unique up to a proportional scaling factor;

- fixed, requiring that the measurement scale is unique (or fixed) with the zero point corresponding to a situation of complete absence.

In addition, the range of the variable can be either bounded or unbounded. The range of a variable is characterized by its lower bound $a_{h}$ and its upper bound $b_{h}$. A bounded variable has both a finite lower and a finite upper bound. An unbounded variable, by contrast, has at least one infinite bound. In this paper, when we deal with unbounded variables we always assume that they have an infinite upper bound and a finite lower bound. ${ }^{2}$ With regard to unbounded ratio-scale and fixed health variables, we adopt the assumption throughout this paper that their lower bound $a_{h}$ is zero, which means that these variables take nonnegative values only.

Given a bounded health variable, we can construct a corresponding ill health variable by calculating the shortfall with regard to the maximum. Starting from the (good) health variable $h_{i}$ we define the ill health variable $s_{i}$ by the following transformation:

$$
s_{i} \equiv b_{h}-h_{i}
$$

The vector $s=\left(s_{1}, s_{2}, \ldots, s_{n}\right)$ represents the ill health situation of the population as a whole. The ill health variable is, of course, also bounded, since it has finite lower and upper bounds $a_{s}=0$ and $b_{s}=\left(b_{h}-a_{h}\right)$. Observe moreover that the averages of the two variables are related to one another by the formula $\mu_{s}=b_{h}-\mu_{h}$.

Table 1 gives an overview of the different possibilities along the axes 'measurement scale' versus bounded or unbounded, and provides example(s) of variables encountered in health economics studies. While most examples speak for themselves, some require more explanation. First, type of illness gets both the label bounded and unbounded. In general,

\footnotetext{
${ }^{2}$ Variables with an infinite lower bound and a finite upper bound can be treated similarly. Variables with infinite lower and upper bounds are of a different character.
} 
one might assume there is an unlimited amount of deficiencies, but if one studies specific health conditions, these are likely to be caused by a limited amount of illnesses only. A similar argument is used for the number of illnesses in the cell (fixed, unbounded). Second, as far as we know, there are no examples of ordinal unbounded health variables in health economics, but for completeness we give the example of an ordinal utility function. ${ }^{3}$ Third, (body) temperature is unbounded and measured on a cardinal scale. Different measurement scales such as Fahrenheit or Celsius arbitrarily fix the zero point, and hence give rise to different cardinal representations. ${ }^{4}$ Fourth, the examples in the rows 'ratio-scale' and 'fixed' show that $a_{h}=0$ appears to be a common-sense assumption: one rarely encounters negative weight/length or health care expenditures.

We discuss the consequences of these different possibilities for inequality measurement in more detail in section 5, but it is worth pointing out here that rank-dependent inequality indices cannot be applied to nominal and ordinal health indicators since nominal and ordinal measurement scales do not allow differences between individuals to be compared. This might seem at odds with the large amount of health inequality studies using indicators of the categorical (e.g. self-assessed health) or the binary type (with 0 indicating the absence and 1 the presence of a certain condition, e.g. immunization against measles). If, however, such a variable can be transformed into or proxied by a cardinal variable, it becomes possible to compare these health differences. ${ }^{5}$ Since we can always treat binary variables as if they were cardinal, we deviate from the common practice and classify them as cardinal variables. ${ }^{6}$ From now on we assume cardinal, ratio-scale or fixed measurement scales; we return to nominal and ordinal scales in section 5 .

\section{A family of rank-dependent inequality indices}

In this section, we present the rank-dependent inequality indices that have been used in the literature and define an encompassing family, separately for bounded and unbounded variables. By defining desirable properties on this family, we arrive in subsequent sections at the value judgements underlying each of these indices.

We start with the most popular rank-dependent inequality index, the health $\mathrm{CI} C(h)$ :

$$
C(h)=\frac{2}{n^{2} \mu_{h}} \sum_{i=1}^{n} z_{i} h_{i}
$$

Eq. (2a) clarifies that the health Concentration Index is a normalized sum of weighted health levels, with the weights being determined by the socioeconomic ranks, i.e. $z_{i}=\lambda_{i}-[(n+1) /$ 2] (Kakwani, 1980: 173-178; Wagstaff et al., 1991). Following Shorrocks (1983), Wagstaff et al. (1991) and Clarke et al. (2002) defined the related Generalized health CI $V(h)$ :

\footnotetext{
${ }^{3}$ As explained before, we assume in this paper that unbounded variables have a finite lower bound (see also footnote 2). This means that only ordinal utility functions with a well-defined finite lower bound would qualify. A similar argument should be made for variables measured on a nominal scale.

${ }^{4}$ Note that temperature has a well-defined finite lower bound at zero Kelvin. This is not the case for all cardinal variables; e.g. the linear index predicting an individual's health status calculated by van Doorslaer and Jones (2003) using an ordered probit model of categorical self-reported health responses, does not seem to have a finite lower bound.

${ }^{5}$ For example, van Doorslaer and Jones (2003) have projected the ordinal self-assessed health categories upon the cardinal HUI-scale. In case of a binary 0/1 indicator, one might overcome the ordinal nature by assuming that it expresses the presence of a certain condition in percentage points, i.e. $100 \%$ or $0 \%$. While this seems somewhat implausible at the individual level, it makes sense at the aggregate level (e.g. percentiles).

${ }^{6}$ On the nature of binary variables, see the recent debate between Wagstaff (2011a,b) and Erreygers and Van Ourti (2011), and also Kjellsson and Gerdtham (2011).
} 


$$
V(h)=\frac{2}{n^{2}} \sum_{i=1}^{n} z_{i} h_{i}
$$

When the health variable is bounded, we can by analogy define the ill health CI $C(s)$ and the Generalized ill health $\mathrm{CI} V(s)$ as:

$$
\begin{gathered}
C(s)=\frac{2}{n^{2} \mu_{s}} \sum_{i=1}^{n} z_{i} s_{i} \\
V(s)=\frac{2}{n^{2}} \sum_{i=1}^{n} z_{i} s_{i}
\end{gathered}
$$

In addition, Wagstaff (2005) and Erreygers (2009a) have developed the Wagstaff Index $W$ $(x)$ and the Erreygers Index $E(x)$, which are indices that can only be applied to bounded health variables. These can be expressed as follows:

$$
\begin{gathered}
W(x)=\frac{2\left(b_{x}-a_{x}\right)}{n^{2}\left(b_{x}-\mu_{x}\right)\left(\mu_{x}-a_{x}\right)} \sum_{i=1}^{n} z_{i} x_{i} \\
E(x)=\frac{8}{n^{2}\left(b_{x}-a_{x}\right)} \sum_{i=1}^{n} z_{i} x_{i}
\end{gathered}
$$

where $x=h, s$.

Equations (2)-(5) are all variants of a general expression which differ only with respect to the normalization applied to the weighted sum of health (c.q. ill health) levels. We define the family of rank-dependent indices by the expression:

$$
I(x)=f\left(a_{x}, b_{x}, \mu_{x}, n\right) \sum_{i=1}^{n} z_{i} x_{i}
$$

where $f($.$) . is a continuous function. For unbounded variables, for which we have b_{x}=+\infty$, the function can be simplified to $f\left(a_{x}, \mu_{x}, n\right)$. This family resembles the class of linear measures of income inequality suggested by Mehran (1976) that are all based on "...a weighted area between the Lorenz curve and the line of perfect equality" (ibidem: 806). ${ }^{7}$

\section{Desirable properties of rank-dependent inequality indices}

The form of the function $f($.$) in (6a) is on purpose very general. As explained before, the$ idea is to make it more specific by looking at the properties which we want the index $I(x)$ to have. This section introduces desirable properties for unbounded and bounded variables and

\footnotetext{
${ }^{7}$ The class of Mehran measures is quite broad; we limit ourselves to the case where the weights $z i$ are determined by a linear function of the socioeconomic ranks. The extended CI examined by Wagstaff (2002) is an example of a Mehran measure not included in our family.
} 
discusses the implications for the family of rank-dependent inequality indices defined by (6a) (for readability, all proofs are provided in the appendix). We start by describing the Sign Condition and Scale Invariance which are relevant for bounded and unbounded variables. Next, we cover the Mirror property, Absolute and Relative Inequality for bounded variables, the Convergence property, and Linearity.

\subsection{Sign Condition}

By convention, positive values of a rank-dependent index are seen as signs of a pro-rich bias in the distribution, negative values as signs of a pro-poor bias, and zero as typical for a distribution which is neither pro-rich nor pro-poor. In order to distinguish these cases, we have to identify the situations in which we want $I(x)$ to indicate that there is no systematic bias in favour of either the rich or the poor. From (6a) it follows that the index will be zero in just two cases: $f()=$.0 and $\sum_{i=1}^{n} z_{i} x_{i}=0$. The case $\sum_{i=1}^{n} z_{i} x_{i}=0$ corresponds to a situation where all persons have the same level of health (or ill health), or to a situation where the health differences between persons are not systematically in favour of either the rich or the poor. These are clearly situations in which we want our index to be zero. But what about the case $f()=$.0 ? Suppose there exists a combination $\left(a_{x}^{0}, b_{x}^{0}, n^{0}, \mu_{x}^{0}\right)$ such that $f\left(a_{x}^{0}, b_{x}^{0}, n^{0}, \mu_{x}^{0}\right)=0$ and $\sum_{i=1}^{n} z_{i} x_{i} \neq 0$. If that were the case, then given $a_{x}^{0}, b_{x}^{0}$ and $n^{0}$ we would always have $I(x)=0$ for any distribution $x$ with the specific mean $\mu_{x}^{0}$. But clearly some of these distributions would be entirely pro-rich and others entirely pro-poor, so that for these we should have $I(x) \neq 0$. This means that we have to ensure that $f()=$.0 can occur only when we have $\sum_{i=1}^{n} z_{i} x_{i}=0$. The easiest way to do so is to assume that $f($.) never changes sign and is different from zero whenever $\sum_{i=1}^{n} z_{i} x_{i} \neq 0.8$ The following condition formalizes this assumption.

Sign Condition-The sign of $I(x)$ coincides with the sign of $\sum_{i=1}^{n} z_{i} x_{i}$.

The Sign Condition obviously leads to the following proposition:

Proposition 1: A rank-dependent index I(x) satisfies the Sign Condition if and only if:

i. $\quad f\left(a_{x}, \mu_{x}, n\right)>0$ for $n>0$ and $a_{x}<\mu_{x}<+\infty$ when $x$ is an unbounded variable;

ii. $f\left(a_{x}, b_{x}, \mu_{x}, n\right)>0$ for $n>0$ and $a_{x}<\mu_{x}<b_{x}$, when $x$ is a bounded variable.

\subsection{Scale Invariance}

The requirement of Scale Invariance is that we want our index to be independent of the unit of measurement of health (or ill health). We consider both cardinal and ratio-scale, and bounded and unbounded variables, but not 'fixed' variables since these have a fixed unit of measurement. Below, we define the requirement, and derive the consequences for the set of admissible indices that belong to the family of rank-dependent inequality indices defined by (6a).

Scale Invariance-Consider a change in the unit of measurement which transforms the distribution $x$ into $\tilde{x}$, the lower bound $a_{x}$ into $\tilde{a}_{x}$, and the upper bound $b_{x}$ into $\tilde{b}_{x}$, either by

\footnotetext{
${ }^{8}$ This allows for $f()=$.0 in the cases $\mu_{x}=a_{x}$ and $\mu_{x}=b_{x}$, since these can occur only if all individuals have the same level $x_{i}$, which implies that $\sum_{i=1}^{n} z_{i} x_{i}=0$.
} 
means of a positive linear transformation (i.e. $\tilde{x}_{i}=\alpha+\beta x_{i}, \tilde{a}_{x}=\alpha+\beta a_{x}$ and $\tilde{b}_{x}=\alpha+\beta b_{x}$, where $\beta>0$ ), when $x$ is a cardinal variable, or by means of a positive proportional transformation (i.e. $\tilde{x}_{i}=\beta x_{i}, \tilde{a}_{x}=\beta a_{x}$ and $\tilde{b}_{x}=\beta b_{x}$, where $\beta>0$ ), when $x$ is a ratio-scale variable. This change does not affect the value of the inequality index, i.e. $I(\tilde{x})=I(x)$.

The requirement of Scale Invariance substantially reduces the set of allowable $f($. expressions.

Proposition 2: A rank-dependent index I (x) has the Scale Invariance property if and only if:

i. $f\left(a_{x}, \mu_{x}, n\right)=\frac{1}{\mu_{x}-a_{x}} k(n)$ when $\mathrm{x}$ is an unbounded variable;

ii.

$$
f\left(a_{x}, b_{x}, \mu_{x}, n\right)=\frac{1}{b_{x}-a_{x}} g\left(\frac{\mu_{x}-a_{x}}{b_{x}-a_{x}}, n\right) \text { when } \mathrm{x} \text { is a bounded variable. }
$$

For unbounded cardinal and ratio-scale variables the set of allowable $f($.) expressions satisfying the Sign Condition and Scale Invariance seems rather narrow. It is so narrow, in fact, that all that is needed to arrive at a unique index is to choose the desired maximum bounds of the index. If these are fixed at -1 and +1 , we are led to the expression $k(n)=2 / n^{2}$ and arrive at the following slightly modified version of the standard CI: ${ }^{9}$

$$
\widehat{C}(x)=\frac{2}{n^{2}\left(\mu_{x}-a_{x}\right)} \sum_{i=1}^{n} z_{i} x_{i}
$$

We believe this is a powerful result that might help to clarify the subtleties of measuring inequalities in the health sector when the health variable is unbounded (see table 1 for some examples). We ended section 2 by stressing that rank-dependent indices cannot be applied to variables measured with a nominal or ordinal scale since differences between individuals are meaningless in those cases. Taking into account the Sign Condition and Scale Invariance we now find that the standard $\mathrm{CI}$ (or, to be precise, $\hat{C}(x)$ ) emerges as the natural candidate when dealing with unbounded ratio-scale and cardinal variables. For unbounded variables measured on a fixed scale, Scale Invariance is irrelevant, and the Sign Condition by itself puts too little structure upon $f($.) to yield a unique index. Nevertheless, fixing the bounds of the index at -1 and +1 and imposing that the index should be 'relative' (i.e., inequality remains constant for any equiproportional change) leads to a unique index: for unbounded variables measured on a fixed scale, the standard $\mathrm{CI}$ is the only relative index. If the index should be 'absolute' rather than 'relative' (i.e., adding the same amount to everyone's level leaves inequality unchanged), the road leads to the so-called Generalized $\mathrm{CI}, \mu_{x} C(x)$, or a scalar multiple of it. ${ }^{10}$

While the Sign Condition and Scale Invariance (or the absolute/relative inequality criterion) manage to sufficiently reduce the family of rank-dependent inequality indices for unbounded variables, the same does not hold for bounded variables. Although some popular indices -

${ }^{9}$ Strictly speaking, the correct expression is $k(n)=\frac{2}{n(n-1)}$; for $k(n)=2 / n^{2}$, the bounds are $(1-n) / n$ and $(n-1) / n$ instead of -1 and +1 . Observe that if $x$ is a ratio-scale variable, we have $a_{x}=0$ and therefore $\hat{C}(x)=C(x)$.

${ }^{10}$ Since for unbounded variables measured on a fixed scale 'relative' is mathematically equivalent to 'scale-invariant', the case for the standard CI follows from Proposition 2. The case for the Generalized CI follows from the fact that $f($.$) must be independent of \mu_{x}$ : for an absolute index we must have $f\left(0, \mu_{x}, n\right)=f\left(0, \mu_{x}+\Delta, n\right)$ with $\Delta>0$ for all possible values of $\mu_{x}$. 
e.g. the standard and generalized CI - are ruled out by Scale Invariance, the set of allowable $f($.) expressions for bounded variables remains very large. In the subsequent sections, we will dig deeper into the family of rank-dependent indices for bounded variables.

Before introducing additional conditions that reduce the set of allowable $f($.$) expressions for$ bounded variables, we highlight an expositional advantage of the condition of Scale Invariance. Since Scale Invariance allows the unit of measurement to be chosen freely, we find it more convenient to represent the health variable (and likewise the ill health variable) in the form suggested by condition (ii) of Proposition 2. The standardized representation of a health variable $h_{i}$ varying between $a_{h}$ and $b_{h}$ is equal to $h_{i}^{*}$ which is defined as:

$$
h_{i}^{*} \equiv \frac{h_{i}-a_{h}}{b_{h}-a_{h}}
$$

This variable is bounded between $a_{h^{*}}=0$ and $b_{h^{*}}=1$. The corresponding standardized ill health variable is then $s_{i}^{*} \equiv\left(b_{h}-h_{i}\right) /\left(b_{h}-a_{h}\right)=1-h_{i}^{*}$. Observe furthermore that $\mu_{h^{*}}=\left(\mu_{h}-\right.$ $\left.a_{h}\right) /\left(b_{h}-a_{h}\right)$ and $\mu_{s^{*}}=\left(b_{h}-\mu_{h}\right) /\left(b_{h}-a_{h}\right)=1-\mu_{h^{*}}$. This representation has the advantage of recording the 'real' changes in health and ill health, but not the 'nominal' ones which are purely the effect of moving from one unit of measurement to another. ${ }^{11}$ The standardized variables can be interpreted as the net health and ill health ratios, because they measure the distance from respectively the lower and the upper bounds, and express it as a proportion of the maximum distance. Hence, the standardized representation allows us to derive results which are valid for three types of bounded variables (cardinal, ratio-scale, and fixed-scale). With this standardized notation, the expression for scale-invariant rank-dependent indices for bounded variables is ${ }^{12}$ :

$$
I\left(x^{*}\right)=g\left(\mu_{x^{*}}, n\right) \sum_{i=1}^{n} z_{i} x_{i}^{*}
$$

\subsection{The Mirror property}

A third requirement is that we would like the health index to be the reflection of the ill health index. ${ }^{13}$ The basic idea is that the ranking of distributions generated by the health index should be the reverse of the ranking generated by the ill health index. Here we consider a rather strong form of this requirement, but we admit that much can be said for considering a weaker form. Such an approach has been followed by Lambert and Zheng (2011), in a slightly different framework; the important lesson from their work is that although they start from indices which satisfy the weak version, they end up with indices which satisfy the strong version. This suggests that nothing much is lost if we immediately work with the strong form of the requirement. The Mirror property which we consider here is formally defined as follows:

\footnotetext{
${ }_{11}$ A change from $x_{i}^{*}$ to $r x_{i}^{*}$ in standardized terms corresponds to a change from level $x_{i}$ to $r x_{i}+(1-r) a_{x}$.

${ }^{12}$ For the three indices that were mentioned previously, the standardized versions of the normalization functions $g\left(\mu_{x}^{*}, n\right)=2 /$ $\left(n^{2} \mu_{x}{ }^{*}\right)$ for $\hat{C}\left(x^{*}\right) ; g\left(\mu_{x}^{*}, n=2 /\left(n^{2} \mu_{x}{ }^{*}\left(1-\mu_{x}^{*}\right)\right)\right.$ for $W\left(x^{*}\right) ; g\left(\mu_{x}^{*}, n=8 / n^{2}\right.$ for $E\left(x^{*}\right)$. Observe that all three normalization functions have the property of multiplicative separability, i.e. they can be expressed $g\left(\mu_{x}^{*}, n\right)=k(n) q\left(\mu_{x}^{*}\right)$. This seems a natural assumption to make, but it is not strictly necessary.

${ }^{13}$ See also Sen (1992: 89-93) for the related distinction between shortfall and attainment inequality for one-dimensional inequality measures.
} 
Mirror-Let $h$ be a given health distribution and $s$ its associated ill health distribution. Then the health index $I(h)$ and the ill health index are equal in absolute value but have opposite signs, i.e. $I(h)=-I(s)$.

For the class of scale-invariant indices in (6b), the Mirror property imposes further structure upon the normalization function $g\left(\mu_{x}{ }^{*} n\right)$ :

Proposition 3: A scale-invariant rank-dependent index I ( $\left.\mathrm{x}^{*}\right)$ has the Mirror property if and only if $\mathrm{g}\left(\mu_{x}^{*}, \mathrm{n}\right)=\mathrm{g}\left(1-x^{*}, \mathrm{n}\right)$.

In mathematical terms, the function $g\left(\mu_{x}^{*}, n\right)$ must be symmetrical around $\mu_{x}{ }^{*}=0.5$ for a given value of $n$. It turns out that the Mirror property is closely related to the elasticity of the normalization function with respect to the mean. Let $\varepsilon\left(\mu_{x^{*}}\right) \equiv \frac{\partial g\left(\mu_{x^{*}}, n\right)}{\partial \mu_{x^{*}}} \frac{\mu_{x^{*}}}{g\left(\mu_{x^{*}}, n\right)}$ denote the elasticity of the normalization function $g\left(\mu_{x}^{*}, n\right)$ with respect to the mean $\mu_{x}{ }^{*}$.

Proposition 4: For a scale-invariant rank-dependent index I(x*) the Mirror property holds if and only if $\varepsilon\left(1-\mu_{x^{*}}\right)=-\frac{\left(1-\mu_{x^{*}}\right)}{\mu_{x^{*}}} \varepsilon\left(\mu_{x^{*}}\right)$.

The Mirror property restricts the set of normalization functions rather drastically; among others it eliminates $\hat{C}\left(x^{*}\right)$, but not $E\left(x^{*}\right)$ and $W\left(x^{*}\right) .{ }^{14}$ In subsequent sections, we will show that the value of the elasticity of the normalization function turns out to be crucial to further characterize the properties of the remaining set of indices.

\subsection{Absolute and relative inequality for bounded variables}

Wagstaff (2009) and Erreygers (2009b) discussed the concepts of absolute and relative inequality extensively. One of the major insights emerging from their discussion is that one cannot impose the meaning of absolute and relative inequality - in the way these have been understood for unbounded ratio-scale variables - upon bounded variables. Our purpose here is to explore these issues in a more formal way.

It may be useful to briefly recall the notions of relative and absolute inequality indices in the case of unbounded ratio-scale variables, such as income (see for example Kolm, 1976). A relative inequality index is an index of which the value remains constant for any proportional change of all individual levels. For unbounded ratio-scale variables, the notion of a relative inequality index therefore coincides with that of a scale-invariant index. An absolute inequality index, by contrast, is characterized by the property of translationinvariance: adding the same amount to everyone's level leaves the value of the inequality index unchanged. The difficulty when trying to apply these notions to bounded variables is that some of the changes are infeasible, because the bounds of the variables act as constraints. This means that we have to adapt the definitions. In order to make the distinction clear, we will for bounded variables refer to 'quasi-relative' and 'quasi-absolute' indices.

Since we are interested in scale-invariant indices only, we stick to the previously introduced standardized representation of health and ill health variables. A scale-invariant rankdependent health (c.q. ill health) inequality index is said to be quasi-relative if it is insensitive to any feasible proportional change of all standardized health (c.q. ill health)

\footnotetext{
${ }^{14} \hat{C}\left(x^{*}\right)$ does not satisfy the condition of Proposition 4 since $\varepsilon\left(\mu_{x}^{*}\right)=\varepsilon\left(1-\mu_{x}^{*}\right)=-1$, but $E\left(x^{*}\right)$ and $W\left(x^{*}\right)$ do, since $\varepsilon\left(1-\mu_{x}^{*}\right)=$ $\varepsilon\left(\mu_{x}^{*}\right)=0$ for $E\left(x^{*}\right)$, and $\varepsilon\left(1-\mu_{x}^{*}\right)=\left(1-2 \mu_{x}^{*}\right) / \mu_{x}^{*}$ and $\varepsilon\left(\mu_{x}^{*}\right)=-\left(1-2 \mu_{x}^{*}\right) /\left(1-\mu_{x}^{*}\right)$ for $W\left(x^{*}\right)$.
} 
levels. In other words, a quasi-relative index takes into account only the relative positions of individuals, not the absolute differences among persons. ${ }^{15}$. Formally, we have:

Quasi-Relativity—Consider a change from $x^{*}$ to $y^{*}$, where $y_{i}^{*}=r x_{i}^{*}$ and $r \neq 0$. If for any feasible $x^{*}$ and $y^{*}$ we have $I\left(y^{*}\right)=I\left(x^{*}\right)$, then $I\left(x^{*}\right)$ is a quasi-relative index.

By analogy we define the notion of a quasi-absolute index:

Quasi-Absoluteness-Consider a change from $x^{*}$ to $y^{*}$, where $y_{i}^{*}=x_{i}^{*}+\Delta$ and $\Delta \neq 0$. If for any feasible $x^{*}$ and $y^{*}$ we have $I\left(y^{*}\right)=I\left(x^{*}\right)$, then $I\left(x^{*}\right)$ is a quasi-absolute index.

The property of Quasi-Absoluteness coincides with the property of Level Independence (Erreygers, 2009a). We now show a number of formal results on quasi-relative and quasiabsolute indices.

Proposition 5: A scale-invariant rank-dependent index I $\left(x^{*}\right)$ is quasi-relative if and only if $\varepsilon\left(\mu_{x}^{*}\right)=-1$.

Proposition 6: A scale-invariant rank-dependent index I $\left(x^{*}\right)$ is quasi-absolute if and only if $\varepsilon\left(\mu_{x}^{*}\right)=0$.

Propositions 4, 5 and 6 suggest that the elasticity $\varepsilon\left(\mu_{x}^{*}\right)$ of the normalization function $g$ $\left(\mu_{x}^{*}, n\right)$ with respect to the mean $\mu_{x}{ }^{*}$ can be used to construct a measure of an index's sensitivity to (quasi-)relative and (quasi-)absolute inequality. We define the weight which an index gives to relative inequality as $-\varepsilon\left(\mu_{x}^{*}\right)$ and the weight which it gives to absolute inequality as $1+\varepsilon\left(\mu_{x}^{*}\right)$. Indices for which both weights are positive are called 'mixed inequality' indices. Indices for which the relative inequality weight is negative and the absolute inequality weight greater than 1 are called 'inverse-relative' indices. These indices have the property of increasing in magnitude when a change occurs which leaves all absolute differences the same and decreases all relative differences. Likewise, indices for which the absolute inequality weight is negative and the relative inequality weight greater than 1 are called 'inverse-absolute' indices. Such indices have the property of increasing in magnitude when a change occurs which leaves all relative differences the same and decreases all absolute differences.

Intuitively, it seems hard to make a case for inverse-relative or inverse-absolute indices, since they move in opposite directions from what we expect. Our choice is therefore confined to mixed inequality indices, with the quasi-relative and quasi-absolute indices as limit cases. It is easy to verify that $\hat{C}\left(x^{*}\right)$ is a quasi-relative index. We have already shown that this index fails the Mirror property. This is in fact not a coincidence, but a general result:

Proposition 7: A scale-invariant rank-dependent index I $\left(\mathrm{x}^{*}\right)$ cannot simultaneously have the properties of Mirror and Quasi-Relativity.

In other words, by restricting ourselves to indicators which have the property of Scale Invariance, we have to choose between Quasi-Relativity and Mirror. If we impose QuasiRelativity, we cannot have Mirror, and we reduce the set of allowable rank-dependent indices to $\hat{C}\left(x^{*}\right)$ or scalar multiples of it (by fixing the bounds of the index at -1 and +1 we

\footnotetext{
${ }^{15}$ A feasible move from $x_{i}^{*}$ to $r x_{i}^{*}(r \neq 0, r \neq 1)$ for all individuals does not change the relative positions $\left(x_{i}^{*} / x_{j}^{*}=r x_{i}^{*} / r x_{j}^{*}\right)$, but changes the absolute differences $\left(\left|x_{i}^{*}-x_{j}^{*}\right| \neq\left|r x_{i}^{*}-r x_{j}^{*}\right|\right.$ for $x_{i}^{*} \neq x_{j}^{*}$ ).
} 
end up with $\left.\hat{C}\left(x^{*}\right)\right) .{ }^{16}$ If, on the other hand, we impose Mirror, we have to relinquish QuasiRelativity, but we do not reduce the set of allowable indices to a unique index. We use the following result to narrow down the set.

Proposition 8: A scale-invariant rank-dependent index I ( $\left.x^{*}\right)$ which has the Mirror property can be of the mixed inequality type for only half of the distributions.

It turns out that when an index is of the mixed inequality type for distributions with mean $\mu_{x}{ }^{*}$, it is inverse-relative for distributions with mean $1-\mu_{x}{ }^{*}$. Given our choice to rule out inverse-relative indices, we are obliged to leave mixed inequality type indices out of consideration. Hence, Proposition 8 implies that we must rule out the Wagstaff index and all other mixed inequality type indices on the grounds that these are inverse-relative for a considerable amount of distributions. ${ }^{18}$ Having ruled out quasi-relative and mixed inequality indices, we are left with only one option: quasi-absolute indices. Here, at last, no further difficulties arise:

Proposition 9: A scale-invariant rank-dependent index $\mathrm{I}\left(\mathrm{x}^{*}\right)$ which has the Mirror property and is never inverse-relative or inverse-absolute must be a quasi-absolute index.

Proposition 9 reduces the set of admissible indices defined by (6b) in essence to a singleton: the only quasi-absolute index of this class is the Erreygers index or scalar multiples of it (assuming that the maximum bounds are -1 and +1 yields $\left.E\left(x^{*}\right)\right) .{ }^{18}$

\subsection{Discussion}

In the previous section we have shown that $\hat{C}\left(x^{*}\right)$ violates the Mirror condition, and that mixed inequality type indices are inverse-relative for some distributions. Whereas the violation of the Mirror condition seems rather obvious, an example may be useful to clarify when mixed inequality type indices are inverse-relative. Since the Wagstaff index is a wellknown mixed inequality index, we select $W\left(x^{*}\right)$ to illustrate the property of inverserelativity, and compare it with the indices which have the property of respectively quasirelativity and quasi-absoluteness, viz. $\hat{C}\left(x^{*}\right)$ and $E\left(x^{*}\right)$. Suppose we start from the distribution $x^{*}=(0.5,0.6,0.7)$, where person 1 is the least well-off and person 3 the best well-off. Since we have $n=3, \mu_{x}{ }^{*}=0.6$ and $\sum_{i=1}^{3} z_{i} x_{i}^{*}=0.2$, it follows that $\hat{C}\left(x^{*}\right)=2 / 27, W$ $\left(x^{*}\right)=5 / 27$ and $E\left(x^{*}\right)=8 / 45$. Now let us add $\Delta=0.2$ to everyone's level, so that the distribution becomes $y^{*}=(0.7,0.8,0.9)$. All absolute differences remain the same, and all relative differences decrease: in relative terms persons 1 and 2 come closer to one another (they move from ratio 5/6 to ratio 7/8), but so do persons 1 and 3 (from 5/7 to 7/9) and persons 2 and 3 (from 6/7 to 8/9). Since we have $n=3, \mu_{y}{ }^{*}=0.8$ and $\sum_{i=1}^{3} z_{i} y_{i}^{*}=0.2$, it follows that $\hat{C}\left(y^{*}\right)=1 / 18, W\left(y^{*}\right)=5 / 18$ and $E\left(y^{*}\right)=8 / 45$. In other words, the modified CI decreases, the Wagstaff index increases, and the Erreygers index remains the same.

Although the value judgments underlying Propositions 7, 8 and 9 should be clear by now, it may be useful to look at the issue from another point of view so as to gain additional insights into the value judgments underlying the different subclasses of the family of scaleinvariant rank-dependent indices defined by (6b). Such an alternative perspective consists of examining what happens when a given unequal distribution is gradually reduced to a perfectly equal distribution. More particularly, it seems interesting to determine the limit value of an index when all individual levels are reduced to zero by means of a proportional

\footnotetext{
16 This is easily seen from noting that $\varepsilon\left(\mu_{x}^{*}\right)=-1$ requires that $g($.$) is homogenous of degree -1$ in $\mu_{x}{ }^{*}$.

${ }^{18}$ This is easily seen from Proposition 6 as $\varepsilon\left(\mu_{x}^{*}\right)=0$ requires the normalization function to be independent of $\mu_{x}{ }^{*}$.
} 
reduction. When all individuals have zero, the distribution is equal and so the value of the index should tend to zero. This is what we call Convergence:

Convergence-Let $x^{*}$ be a given distribution. Consider a change which reduces everyone's position from $x_{i}^{*}$ to $r x_{i}^{*}$. Then $\lim _{r \rightarrow 0} I\left(r x^{*}\right)=0$.

The following result relates the property of Convergence to the normalization function.

Proposition 10: A scale-invariant rank-dependent index $\mathrm{I}\left(\mathrm{x}^{*}\right)$ has the property of Convergence if and only if $\lim _{\mu_{x^{*}} \rightarrow 0} g\left(\mu_{x^{*}}, n\right) \mu_{x^{*}}=0$.

Convergence reduces the set of admissible normalization functions considerably. It turns out that it is not satisfied by the only scale-invariant and quasi-relative index (the modified CI), and neither by a subset of mixed inequality type indices, including the Wagstaff Index. This is straightforwardly illustrated for the normalization functions of the modified $\mathrm{CI}$ and the Wagstaff Index since these have the property of multiplicative separability: they can be written as $g\left(\mu_{x}^{*}, n\right)=k(n) q\left(\mu_{x}^{*}\right)$, where $k(n)=2 / n^{2}$ and $q\left(\mu_{x}{ }^{*}\right)=\mu_{x}{ }^{*}-1$ for the modified CI and $q\left(\mu_{x}^{*}\right)=\left[\mu_{x}^{*}\left(1-\mu_{x}^{*}\right)\right]^{-1}$ for the Wagstaff Index. Hence, we have for both indices that $\lim _{\mu_{x^{*}} \rightarrow 0} g\left(\mu_{x^{*}}, n\right) \mu_{x^{*}}=2 / n^{2}$, which means that the condition of Proposition 10 does not hold. The reason why a subset of mixed inequality type indices (including the Wagstaff Index) do not have the property of Convergence is that these indices cease to attach any weight to quasiabsolute inequality when the value of $\mu_{x}{ }^{*}$ approaches 0 (in other words, similarly to the modified CI, they have $\varepsilon(0)=-1)$.

The Convergence property looks at the limit value of the index when there is an equiproportional reduction of all individual levels. Even if the limit value of an index tends to 0 , many different types of trajectories remain possible. A linear trajectory seems simple: it implies that when all individual levels are halved, then the measured degree of inequality is also cut in half. This leads to the following property and proposition:

Linearity-Let $x^{*}$ be a given distribution. Consider a change which reduces everyone's position from $x_{i}^{*}$ to $r x_{i}^{*}$, with $0 \leq r<1$. Then we have: $I\left(r x^{*}\right)=r I\left(x^{*}\right)$

Proposition 11: A scale-invariant rank-dependent index $\mathrm{I}\left(\mathrm{x}^{*}\right)$ has the property of Linearity if and only if $\mathrm{g}\left(\mu_{x}^{*}, \mathrm{n}=\mathrm{k}(\mathrm{n})\right.$.

It is easy to check that of all the indices considered so far, the Erreygers index is the only one satisfying the property of Linearity.

\section{Properties and measurement scale of health variables and implications for the class of rank-dependent inequality indices}

We have previously discussed the measurement scales of health variables and presented an overview with examples in table 1 . The purpose of this section is to link these measurement scales to the properties discussed in the previous sections in order to provide guidelines to discriminate between the rank-dependent inequality indices. These guidelines are summarized in table 2 .

Recall that we have differentiated health variables (and by extension health care and expenditure indicators) along two axes, i.e. (i) health variables can be bounded or unbounded, and (ii) the measurement scale can be nominal, ordinal, cardinal, ratio-scale, or 
fixed. We explained in section 2 that rank-dependent inequality indices cannot be applied to nominal and ordinal health indicators since differences between individuals are meaningless with those measurement scales. More generally, we do not see how one could meaningfully measure inequalities in nominal health indicators (using rank-dependent indices, other classes of indices, dominance relations or other measurement frameworks) as these indicators do not rank health states. By contrast, ordinal health indicators might be subjected to inequality measurement. While rank-dependent inequality indices cannot be applied directly to the raw ordinal health indicators, applied health inequality researchers have projected cardinal scales upon these ordinal health indicators, circumventing the meaninglessness of ordinal health differences (for example, van Doorslaer and Jones, 2003; see also section 2). Although in the health inequality literature the projections have until now always generated cardinally scaled variables, they can in principle also lead to ratioscaled variables. This method solves the incompatibility between rank-dependent inequality indices and ordinal health differences, but that does not mean that any rank-dependent inequality index can be used. In view of our findings on Scale Invariance in section 4.2, one should apply the modified CI when the projection gives rise to an unbounded cardinal variable, the $\mathrm{CI}$ when the projection leads to an unbounded ratio-scale variable, and any member of the class of indices defined in (6b) when the projection generates a bounded cardinal or ratio-scale variable. ${ }^{19}$

The property of Scale Invariance is in our view indispensable as it makes the value of an index independent of the specific unit in which a variable is expressed. Scale-invariant indices allow to compare inequality levels of variables with different cardinal representations (for example inequality in health care expenditures versus inequality in the health utility index). For unbounded variables, Scale Invariance has important implications for the kind of inequality that can be measured. If the variable is of the cardinal or ratioscale type, one can only meaningfully measure relative inequalities. Measuring absolute inequalities using the Generalized CI is only feasible for unbounded indicators with a fixed scaling.

For bounded variables, whether they be of the cardinal, ratio- or of the fixed-scale type, the choice narrows down to the class of indices defined by (6b). What index to choose from this subset depends on one's judgements on how the rank-dependent inequality index should react to changes in average standardized health (or ill health). ${ }^{20}$ We have presented two equivalent ways to characterize the properties of this subset of indices.

The first starts from the concepts of absolute and relative inequality. These concepts have a well-known meaning for unbounded variables such as income. Relative inequality indices are insensitive to equiproportional changes, whereas absolute inequality indices are insensitive to equal additions. However, these concepts are difficult to apply to bounded variables since for many distributions equiproportional changes or equal additions are infeasible due to the bounds of the variables acting as constraints. As a result, we have introduced the notions of quasi-relative and quasi-absolute inequality which state that a rank-dependent inequality index should be insensitive to any feasible equiproportional change (Quasi-Relativity) or any feasible equal addition (Quasi-Absoluteness) of the

\footnotetext{
${ }^{19}$ Alternatively, one could desert the class of rank-dependent inequality indices and resort to other measurement approaches that take the ordinal nature of the health indicators explicitly into account. Allison and Foster (2004) have developed a median-based partial inequality ordering for ordinal health variables for the measurement of pure health inequalities. Abul Naga and Yalcin (2008) have extended this approach to inequality indices (see Madden (2009) for an application). Apouey (2007) has proposed a class of health polarization indices that is also median-based. Erreygers (2009c) has explored an Atkinson approach to the measurement of socioeconomic health inequalities, and Zheng (2006) has developed dominance conditions to evaluate bivariate health-income distributions that are free from any cardinal valuation of health status.

${ }^{20}$ Note that this standardized representation is also an easy way out if individuals have different minimum and maximum values of the health indicator (for example, it is common to use different threshold levels for the BMI of males and females).
} 
standardized variables. It is essential that these notions are stated in terms of the standardized representation in order to rule out the impact of nominal changes which are purely the effect of moving from one unit of measurement to another. We have shown in section 4.4 that the indices belonging to the class defined by (6b) cannot simultaneously have the properties of Mirror and Quasi-Relativity, entailing an inescapable choice: by stressing Quasi-Relativity, the modified CI emerges as the only index, while imposing Mirror leads to the subset of indices defined by (6b) satisfying the Mirror property. We have shown that the Erreygers Index is the only rank-dependent inequality measure that has the properties of Mirror and Quasi-Absoluteness. All other indices belonging to the class defined by (6b) which have the Mirror property, including the Wagstaff index, turn out to be partly 'inverse-relative'. We believe it is far from obvious to justify the use of inverserelative indices, since they increase in magnitude when there is a change that decreases relative differences while keeping absolute differences the same.

An alternative characterization of the properties of the subset of indices defined by (6b) was based on the idea that a proportional reduction of the standardized health (cf. ill health) levels of all individuals should in the limit reduce the value of the index to zero. The modified CI and a subset of the indices satisfying Mirror (including the Wagstaff index) do not have this property. The Erreygers Index differs from others indices which do have this property in that it moves to this situation of perfect equality in a linear way.

\section{Discussion and conclusion}

The title of this paper reflects our goal of providing guidelines to applied health inequality researchers on the suitability of various rank-dependent inequality indices when dealing with health, health care, and health expenditure indicators with different properties and measurement scales. In order to come up with these guidelines, we have formally proven a set of propositions with regard to properties which we think are relevant.

We differentiated these indicators according to two criteria: (i) whether they are bounded or unbounded and (ii) whether they have a nominal, ordinal, cardinal, ratio-scale or fixed measurement scale. We summarize our guidelines in table 2. Our main conclusions are:

- Inequality measurement is meaningless for nominal variables.

- Rank-dependent inequality indices are in principle meaningless for ordinal variables, but this can be circumvented by projection into a cardinal or ratio-scale variable.

- The most popular rank-dependent inequality index - i.e. the CI that measures relative inequalities - can be applied only to unbounded variables with ratio- or fixed scale, while another popular variant - the Generalized CI that measures absolute inequalities - can be applied only to unbounded variables with fixed scale. We have also introduced a Modified CI that can be applied to unbounded variables with cardinal scale.

- For bounded variables with cardinal, ratio-, or fixed scale, we have advocated the modified CI when one deems Quasi-Relativity more important than Mirror, and a class of scale-invariant rank-dependent indices that satisfy the Mirror property. Among this class, the Erreygers Index is the only index measuring quasi-absolute inequality, i.e. it is insensitive to any feasible equal addition to the standardized variable. If one does not want to impose this assumption, one could use the other members of the class of scale-invariant rank-dependent indices (including the Wagstaff index) that satisfy the Mirror property. In this case, one implicitly agrees 
that for some distributions inequality increases in magnitude when there is a ceteris paribus decrease of relative differences.

In addition to providing guidelines, we highlighted the implications of the new properties derived in this paper (and in the work of Wagstaff and Erreygers) for the long-lasting debate in health economics and epidemiology on measuring absolute versus relative health differences. In this literature, it has become common practice to report both absolute and relative health differences as these might rank distributions differently. For example, Mackenbach et al. (2008) report that absolute inequalities in overall mortality rates between lower and higher educated are the highest in Estonia, while relative inequalities are the highest in the Czech Republic and Estonia only ranks fifth in relative terms. More generally, it is traditionally conceived that analyzing relative inequalities is conceptually different from analyzing absolute inequalities and that one should analyze each separately. For example, Harper and Lynch (2007) give the example of using the CI for measuring relative inequalities, and the Generalized CI for measuring absolute inequalities in smoking prevalence, which indeed is a bounded variable. If one accepts the Mirror property - and we believe one should (although it is ultimately a value judgment that readers have to make for themselves) - the findings in this paper show however that the only case in which the CI and the Generalized CI should be combined to measure both absolute and relative inequalities is when health is unbounded and measured on a fixed scale. When the (raw) indicator is bounded, the absolute/relative dichotomy becomes much more subtle for two reasons. First, notions of absolute and relative inequality should only be defined for the standardized representation of health variables to make sure that one is not 'confused' by mere changes in the measurement scale of the health indicator, and to account for the fact that the bounded nature places restrictions on the feasible relative and absolute changes (for example: individuals with maximum health cannot improve their health level). Second and taking the standardized representation for granted, we have shown that quasi-relative indices are incompatible with the Mirror property, but that quasi-absolute are not. As stated by Erreygers (2009b: 3): "Apparently, many people find it hard to accept that the simple change from an unbounded to a bounded variable can make much of a difference. But it does."

Finally, we have to mention a few limitations of our study. First, we have focused on a family of rank-dependent indicators which may be seen as somewhat narrow. We are well aware that the set of potentially interesting indicators is much larger than the family we have considered. Yet we believe that it makes sense to concentrate on this family, because it contains the indices which are used most frequently by practitioners as well as the variants which have been proposed recently in the literature. The indices of this family differ only with respect to their normalization function. Although the choice of the normalization function may seem like a trivial issue - which indeed it is when we compare only distributions with the same number of individuals and the same average health level - it has important implications when we are comparing distributions with different average health levels. According to the chosen normalization, the perceived change of inequality may be positive, negative, or zero, and therefore it is crucial to make a good choice. Second, we have assumed that the minimum and maximum values of the indicator are known. This is a plausible assumption for several indicators (e.g. the number of nights an individual spends in hospital), but might be implausible for others. For example, while it is generally agreed that life expectancy is bounded, it is not exactly clear what the maximum bound might be; and the measured degree of inequality will depend on the value of this maximum bound. A possible way out might be to develop indices that make distributional assumptions on the length of life, much along the lines of Kakwani (1995) who developed a class of poverty measures that takes account of the uncertainty involved in the specification of the poverty line. 


\section{Acknowledgments}

Tom Van Ourti is supported by the National Institute on Ageing, under grant R01AG037398-01, and also acknowledges support from the NETSPAR project "Income, health and work across the life cycle" and "Health and income, work and care across the life cycle II". Part of this research was undertaken when he was a Postdoctoral Fellow of the Netherlands Organisation for Scientific Research - Innovational Research Incentives Scheme - Veni. We would like to thank Gustav Kjellsson, Dennis Petrie, Eddy van Doorslaer, seminar participants at the University of Lund, and two anonymous referees for useful comments on previous drafts of the paper. The usual caveats apply and all remaining errors are our responsibility. While this paper went through the reviewing process, Adam Wagstaff wrote a note in which he criticized our treatment of binary variables. Readers may find it interesting to consult his note (Wagstaff, 2011a), our comment (Erreygers and Van Ourti, 2011) and his reply (Wagstaff, 2011b).

\section{References}

Abul Naga RH, Yalcin T. Inequality measurement for ordered response health data. Journal of Health Economics. 2008; 27:1614-1625. [PubMed: 18838185]

Allison RA, Foster JE. Measuring health inequality using qualitative data. Journal of Health Economics. 2004; 23:505-524. [PubMed: 15120468]

Apouey B. Measuring health polarization with self-assessed health data. Health Economics. 2007; 16(9):875-894. [PubMed: 17705333]

Avendano Pabon M. Smoking and inequalities. The Lancet. 2006; 368:1417-1418.

Bleichrodt H, van Doorslaer E. A welfare economics foundation for health inequality measurement. Journal of Health Economics. 2006; 26:945-957. [PubMed: 16466818]

Bommier A, Stecklov G. Defining health inequality: why Rawls succeeds where social welfare theory fails. Journal of Health Economics. 2002; 21:497-513. [PubMed: 12022270]

Clarke PM, Gerdtham UG, Johannesson M, Bingefors K, Smith L. On the measurement of relative and absolute income-related health inequality. Social Science and Medicine. 2002; 55:1923-1928. [PubMed: 12406461]

Erreygers G. Correcting the concentration index. Journal of Health Economics. 2009a; 28:504-515. [PubMed: 18367273]

Erreygers G. Correcting the concentration index: A reply to Wagstaff. Journal of Health Economics. 2009b; 28:521-524.

Erreygers, G. A Dual Atkinson Measure of Socioeconomic Inequality of Health. Paper presented at the conference 'New Directions in Welfare'; 29 June - 1 July 2009; mimeo. St Catherine's College, Oxford University; 2009c.

Erreygers G, Van Ourti T. Putting the cart before the horse. Comment on "The concentration index of a binary outcome revisited". Health Economics. 2011 forthcoming.

Feeny D, Furlong W, Torrance GW, Goldsmith CH, Zhu Z, DePauw S, Denton M, Boyle M. Multiattribute and single-attribute utility functions for the Health Utilities Index Mark 3 System. Medical Care. 2002; 40(2):113-128. [PubMed: 11802084]

Fleurbaey M, Schokkaert E. Unfair inequalities in health and health care. Journal of Health Economics. 2009; 28(1):73-90. [PubMed: 18829124]

Furlong WJ, Feeny DH, Torrance GW, Barr RD. The Health Utilities Index (HUI) System for assessing health-related Quality of Life in clinical studies. Annals of Medicine. 2001; 33(5):375384. [PubMed: 11491197]

Harper S, Lynch J. Commentary: Using innovative inequality measures in epidemiology. International Journal of Epidemiology. 2007; 36(4):926-928. [PubMed: 17823143]

Harper S, King NB, Meersman SC, Reichman ME, Breen N, Lynch J. Implicit value judgments in the measurement of health inequalities. The Milbank Quarterly. 2010; 88(1):4-29. [PubMed: 20377756]

Kakwani, NC. Income Inequality and Poverty. Methods of Estimation and Policy Applications. Oxford University Press; Oxford: 1980.

Kakwani N. Measuring poverty with uncertain poverty threshold. Journal of Income Distribution. 1995; 5(2):201-209. 
Kjellsson, G.; Gerdtham, UG. Working Paper No 2011. Department of Economics, Lund University; 2011. Correcting the Concentration Index for Binary Variables; p. 4

Kolm SC. Unequal inequalities II. Journal of Economic Theory. 1976; 13:82-111.

Lambert, P. The Distribution and Redistribution of Income. 3. Manchester University Press; Manchester: 2001.

Lambert P, Zheng B. On the consistent measurement of attainment and shortfall inequality. Journal of Health Economics. 2011; 30(1):214-219. [PubMed: 21129796]

Lindelöw M. Sometimes more equal than others: how health inequalities depend on the choice of welfare indicator. Health Economics. 2006; 15:263-279. [PubMed: 16259049]

Mackenbach JP, Kunst AE. Measuring the magnitude of socio-economic inequalities in health: an overview of available measures illustrated with two examples from Europe. Social Science and Medicine. 1997; 44(6):757-771. [PubMed: 9080560]

Mackenbach JP, Stirbu I, Roskam AJR, Schaap MM, Menvielle G, Leinsalu M, Kunst AE. Socioeconomic inequalities in health in 22 European countries. The New England Journal of Medicine. 2008; 358(23):2468-2481. [PubMed: 18525043]

Madden D. Ordinal and cardinal measures of health inequality: an empirical comparison. Health Economics. 2009; 19(2):243-250. [PubMed: 19301418]

Mehran F. Linear measures of income inequality. Econometrica. 1976; 44:805-809.

O’Donnell, O.; van Doorslaer, E.; Wagstaff, A.; Lindelöw, M. Analyzing Health Equity Using Household Survey Data: A Guide to Techniques and Their Implementation. The World Bank; Washington DC: 2008.

Oliver A, Healey A, Le Grand J. Addressing health inequalities. The Lancet. 2002; 360:565-567.

Regidor E. Measures of health inequalities: part 2. Journal of Epidemiology and Community Health. 2004; 58:900-903. [PubMed: 15483304]

Regidor E, Sánchez E, de la Fuente L, Luquero FJ, de Mateo S, Domínguez V. Major reduction in AIDS-mortality inequalities after HAART: The importance of absolute differences in evaluating interventions. Social Science and Medicine. 2009; 68:419-426. [PubMed: 19056156]

Roberts, F. Measurement Theory with Applications to Decisionmaking, Utility, and the Social Sciences. Addison-Wesley; Reading, Massachusetts: 1979.

Sen, A. Inequality Reexamined. Clarendon Press; Oxford: 1992.

Shorrocks AF. Ranking income distributions. Economica. 1983; 50:3-17.

van Doorslaer E, Jones AM. Inequalities in self-reported health: validation of a new approach to measurement. Journal of Health Economics. 2003; 22:61-87. [PubMed: 12564718]

Van Doorslaer, E.; Van Ourti, T. Chapter 36: Measuring inequality and inequity in health and health care. In: Smith, P.; Glied, S., editors. The Oxford Handbook of Health Economics. Oxford University Press; Oxford: forthcoming

Wagstaff A. Inequality aversion, health inequalities, and health achievement. Journal of Health Economics. 2002; 21:627-641. [PubMed: 12146594]

Wagstaff A. The bounds of the concentration index when the variable of interest is binary, with an application to immunization inequality. Health Economics. 2005; 14(4):429-432. [PubMed: 15495147]

Wagstaff A. Correcting the concentration index: A comment. Journal of Health Economics. 2009; 28:516-520. [PubMed: 19167117]

Wagstaff A. The concentration of a binary outcome revisited. Health Economics. 2011a forthcoming.

Wagstaff A. Reply to Guido Erreygers and Tom Van Ourti's comment on "The concentration index of a binary outcome revisited". Health Economics. 2011b forthcoming.

Wagstaff A, Paci P, van Doorslaer E. On the measurement of inequalities in health. Social Science and Medicine. 1991; 33(5):545-557. [PubMed: 1962226]

Wagstaff A, Watanabe N. What difference does the choice of SES make in health inequality measurement? Health Economics. 2003; 12(10):885-890. [PubMed: 14508873]

Zheng, B. Measuring health inequality and health opportunity. Paper presented at the conference Advancing Health Equity; Helsinki: WIDER-UNU; 2006. 


\section{Appendix: Proofs of the Propositions}

\section{Proposition 2}

i. Suppose that $x$ is a distribution of an unbounded cardinal variable, and consider the positive linear transformation which transforms $x$ into $\tilde{x}$, with $\tilde{x}_{i}=\alpha+\beta x_{i}$. Scale invariance means that $I(x)=I(\tilde{x})$ must hold for any $x$, any $\alpha$, and any $\beta>0$. Since $\sum_{i=1}^{n} z_{i} \tilde{x}_{i}=\beta \sum_{i=1}^{n} z_{i} x_{i}=\beta Z$, the equality $I(x)=I(\tilde{x})$ holds if and only if $f\left(a_{x}, \mu_{x}, n\right) Z$ $=\beta f\left(\alpha+\beta a_{x}, \alpha+\beta \mu_{x}, n\right) Z$. Since $Z$ can be positive, negative or zero, we must always have $f\left(a_{x}, \mu_{x}, n\right)=\beta f\left(\alpha+\beta a_{x}, \alpha+\beta \mu_{x}, n\right)$. If we take $\beta>0$ and $\alpha=-\beta a_{x}$, it follows that we must have $f\left(a_{x}, \mu_{x}, n=\beta f\left(0, \beta\left(\mu_{x}-a_{x}\right), n\right)=\beta g\left(\beta\left(\mu_{x}-a_{x}\right), n\right)\right.$. This implies that $\beta^{-1} g\left(\mu_{x}-a_{x}, n\right)=g\left(\beta\left(\mu_{x}-a_{x}\right), n\right)$, i.e. $g($. $)$ is homogeneous of degree -1 in $\left(\mu_{x}-a_{x}\right)$, for $n$ kept fixed. Using Euler's homogeneous function theorem, it follows that $\left.g\left(\mu_{x}-a_{x}, n\right)=\left(\mu_{x}-a_{x}\right)\right]^{-1} k(n)$. This establishes the necessity part. The sufficiency part is shown by noting that a positive linear transformation changes $\left(\mu_{x}-a_{x}\right)^{-1} k(n)$ into $\left[\beta\left(\mu_{x}-a_{x}\right)\right]^{-1} k(n)$ from we which it follows that $I(\tilde{x})=I(x)$.

If $x$ is a distribution of an unbounded ratio-scale variable, we have $a_{x}=0$ and therefore $f\left(a_{x}, \mu_{x}, n\right)=g\left(\mu_{x}, n\right)$. Since only positive proportional transformations are allowed, $I(x)=I(\tilde{x})$ now implies $g\left(\mu_{x}, n\right)=\beta g\left(\beta \mu_{x}, n\right)$, i.e. $g($.$) is$ homogeneous of degree -1 in $\mu_{x}$, for $n$ kept fixed. Again applying Euler's homogeneous function theorem, it follows that $g\left(\mu_{x}, n\right)=\mu x^{-1} k(n)=\mu_{x}^{-1} k(n)$ and necessity is proved. Sufficiency is obvious.

ii. Suppose that $x$ is a distribution of a bounded cardinal variable. Following the same reasoning as before, we derive that $I(x)=I(\tilde{x})$ implies $f\left(a_{x}, b_{x}, \mu_{x}, n\right)=\beta f\left(\alpha+\beta a_{x}\right.$, $\left.\alpha+\beta b_{x}, \alpha+\beta \mu_{x}, n\right)$. For $\alpha=-a_{x} /\left(b_{x}-a_{x}\right)$ and $\beta=1 /\left(b_{x}-a_{x}\right)$ we obtain

$f\left(a_{x}, b_{x}, \mu_{x}, n\right)=\left(b_{x}-a_{x}\right)^{-1} f\left(0,1, \frac{\mu_{x}-a_{x}}{b_{x}-a_{x}}, n\right)=\left(b_{x}-a_{x}\right)^{-1} g\left(\frac{\mu_{x}-a_{x}}{b_{x}-a_{x}}, n\right)$. This establishes the necessity part. The sufficiency part can be checked by inspection: a positive linear transformation has no effect on $\left(\mu_{x}-a_{x}\right) /\left(b_{x}-a_{x}\right)$, and multiplies the value of $b_{x}-a_{x}$ by $\beta$. Hence $f\left(\alpha+\beta a_{x}, \alpha+\beta b_{x}, \alpha+\beta \mu_{x}, n\right)=f\left(a_{x}, b_{x}, \mu_{x}, n\right) / \beta$, and therefore $I(\tilde{x})=I(x)$. If $x$ is a distribution of a ratio-scale variable, necessity and sufficiency are proved in a similar way.

\section{Proposition 3}

For scale-invariant indices the Mirror property holds if and only if $I\left(x^{*}\right)=-I\left(1-x^{*}\right)$ for all $x^{*}$. Since $\sum_{i=1}^{n} z_{i}\left(1-x_{i}^{*}\right)=-\sum_{i=1}^{n} z_{i} x_{i}^{*}=-Z$ and $\mu_{x}^{*}=1-\mu_{x}^{*}$, this holds if and only if $g\left(\mu_{x}^{*}, n\right) Z=g\left(1-\mu_{x}{ }^{*}, n\right) Z$. Since $Z$ can be positive, negative or zero, we must always have $g\left(\mu_{x}^{*}, n\right)=g\left(1-\mu_{x}^{*}, n\right.$.

\section{Proposition 4}

By definition we have $\varepsilon\left(1-\mu_{x^{*}}\right)=\frac{\partial g\left(1-\mu_{x^{*}}, n\right)}{\partial\left(1-\mu_{x^{*}}\right)} \frac{\left(1-\mu_{x^{*}}\right)}{g\left(1-\mu_{x^{*}}, n\right)}$. By the rules of derivation we have $\frac{\partial g\left(1-\mu_{x^{*}}, n\right)}{\partial \mu_{x^{*}}}=\frac{\partial g\left(1-\mu_{x^{*}}, n\right)}{\partial\left(1-\mu_{x^{*}}\right)} \frac{\partial\left(1-\mu_{x^{*}}\right)}{\partial \mu_{x^{*}}}=-\frac{\partial g\left(1-\mu_{x^{*}}, n\right)}{\partial\left(1-\mu_{x^{*}}\right)}$. Because of the Mirror property we have $g\left(\mu_{x}^{*}, n\right)=g\left(1-\mu_{x}^{*}, n\right)$, and we derive that $\frac{\partial g\left(1-\mu_{x^{*}}, n\right)}{\partial \mu_{x^{*}}}=\frac{\partial g\left(\mu_{x^{*}}, n\right)}{\partial \mu_{x^{*}}}$. 
This means that $\varepsilon\left(1-\mu_{x^{*}}\right)=-\frac{\partial g\left(\mu_{x^{*}}, n\right)}{\partial \mu_{x^{*}}} \frac{\left(1-\mu_{x^{*}}\right)}{g\left(1-\mu_{x^{*}}, n\right)}=-\frac{\partial g\left(\mu_{x^{*}}, n\right)}{\partial \mu_{x^{*}}} \frac{\mu_{x^{*}}}{g\left(\mu_{x^{*}}, n\right)} \frac{\left(1-\mu_{x^{*}}\right)}{\mu_{x^{*}}}$, and we obtain $\varepsilon\left(1-\mu_{x^{*}}\right)=-\frac{\left(1-\mu_{x^{*}}\right)}{\mu_{x^{*}}} \varepsilon\left(\mu_{x^{*}}\right)$.

\section{Proposition 5}

Since we have $\sum_{i=1}^{n} z_{i} y_{i}^{*}=\sum_{i=1}^{n} z_{i}\left(r x_{i}^{*}\right)=r \sum_{i=1}^{n} z_{i} x_{i}^{*}=r Z$ and $\mu_{y}{ }^{*}=r \mu_{x}{ }^{*}$, it follows that $I\left(x^{*}\right)-$ $I\left(y^{*}\right)=\left[g\left(\mu_{x}^{*}, n\right)-r g\left(r \mu_{x}^{*}, n\right)\right] Z$. The Quasi-Relativity property holds if and only if $I\left(x^{*}\right)$ $-I\left(y^{*}\right)=0$ for any feasible $x^{*}$ and $y^{*}$. Since $Z$ can be positive, negative or zero, and $r \mu_{x}{ }^{*}$ can in principle take any value between 0 and 1 , this means that we must have $r^{-1} g\left(\mu_{x}^{*}, n\right)$ $=g\left(r \mu_{x}^{*}, n\right)$. In other words, $g\left(r \mu_{x}^{*}, n\right)$ is homogeneous of degree -1 in $\mu_{x}{ }^{*}$, which means we have $\varepsilon\left(\mu_{x}^{*}\right)=1$.

\section{Proposition 6}

Since we have $\sum_{i=1}^{n} z_{i} y_{i}^{*}=\sum_{i=1}^{n} z_{i}\left(x_{i}^{*}+\Delta\right)=\sum_{i=1}^{n} z_{i} x_{i}^{*}+\Delta \sum_{i=1}^{n} z_{i}=\sum_{i=1}^{n} z_{i} x_{i}^{*}=Z$ and $\mu_{y}{ }^{*}=\mu_{x}{ }^{*}+$ $\Delta$, it follows that $I\left(x^{*}\right)-I\left(y^{*}\right)=\left[g\left(\mu_{x}^{*}, n\right)-g\left(\mu_{x}^{*}+\Delta, n\right)\right] Z$. The Quasi-Absoluteness property holds if and only if $I\left(x^{*}\right)-I\left(y^{*}\right)=0$ for any feasible $x^{*}$ and $y^{*}$. Since $Z$ can be positive, negative or zero, and $\mu_{x}{ }^{*}+\Delta$ can in principle take any value between 0 and 1 , this means that $g\left(\mu_{x}^{*}, n\right)$ must be independent of $\mu_{x}^{*}$. In other words, we have $\varepsilon\left(\mu_{x}^{*}\right)=0$.

\section{Proposition 7}

According to Proposition 5 the Quasi-Relativity property holds if and only if $\varepsilon\left(\mu_{x}^{*}\right)=\varepsilon(1-$ $\left.\mu_{x}^{*}\right)=-1$. According to Proposition 4 the Mirror property holds if and only if $\varepsilon\left(1-\mu_{x^{*}}\right)=-\frac{\left(1-\mu_{x^{*}}\right)}{\mu_{x^{*}}} \varepsilon\left(\mu_{x^{*}}\right)$. Hence both conditions can hold simultaneously only if (1$\left.\mu_{x}^{*}\right) / \mu_{x}^{*}=-1$, which is impossible.

\section{Proposition 8}

Let an index be of the mixed inequality type for some $\mu_{x}^{*}$, which means that $-1<\varepsilon\left(\mu_{x}^{*}\right)<$ 0 . If it has the Mirror property, we know from Proposition 4 that $\varepsilon\left(1-\mu_{x^{*}}\right)=-\frac{\left(1-\mu_{x^{*}}\right)}{\mu_{x^{*}}} \varepsilon\left(\mu_{x^{*}}\right)$. Hence $\varepsilon\left(1-\mu_{x}^{*}\right)>0$, which means that the index is inverse-
relative for $1-\mu_{x}^{*}$

\section{Proposition 9}

An index is never inverse-relative or inverse-absolute if and only if $-1 \leq \varepsilon\left(\mu_{x}^{*}\right) \leq 0$ for all 0 $\leq \mu_{x}{ }^{*} \leq 1$. From Proposition 8 we know that if $-1<\varepsilon\left(\mu_{x}^{*}\right)<0$ for some $\mu_{x}{ }^{*}$, then for $1-$ $\mu_{x}{ }^{*}$ the index will be inverse-relative if it has the Mirror property. If $\varepsilon\left(\mu_{x}^{*}\right)=-1$ for all $0 \leq$ $\mu_{x}^{*} \leq 1$, for all $0 \leq \mu_{x}^{*} \leq 1$, then the index does not have the Mirror property. If $\varepsilon\left(\mu_{x}^{*}\right)=0$ for all $0 \leq \mu_{x}{ }^{*} \leq 1$, then the index has the Mirror property and is never inverse-relative or inverse-absolute.

\section{Proposition 10}

Let $y^{*}=r x^{*}$, and assume that $\sum_{i=1}^{n} z_{i} x_{i}^{*}=Z \neq 0$. Since $\sum_{i=1}^{n} z_{i} y_{i}^{*}=r \sum_{i=1}^{n} z_{i} x_{i}^{*}$ and $\mu_{y}{ }^{*}=r \mu_{x}^{*}$, we have $I\left(r x^{*}\right)=g\left(r \mu_{x}{ }^{*}, n\right) r Z=g\left(\mu_{y}{ }^{*}, n\right) \mu_{y}{ }^{*}\left(Z / \mu_{x}{ }^{*}\right)$. Since $Z / \mu_{x}{ }^{*}$ is a constant different 
from zero, the condition $\lim _{r \rightarrow 0} I\left(r x^{*}\right)=0$ is equivalent to the condition $\lim _{\mu_{y^{*}} \rightarrow 0} g\left(\mu_{y^{*}}, n\right) \mu_{y^{*}}=0$. If $Z=$ 0 , we have $I\left(r x^{*}\right)=0$ whatever may be the value of $r$.

\section{Proposition 11}

Let $y^{*}=r x^{*}$, and assume that $\sum_{i=1}^{n} z_{i} x_{i}^{*}=Z \neq 0$. Linearity means we have $I\left(r x^{*}\right)=r I\left(x^{*}\right)$ for any $x^{*}$ and any $0 \leq r \leq 1$. Since $\sum_{i=1}^{n} z_{i} y_{i}^{*}=r \sum_{i=1}^{n} z_{i} x_{i}^{*}$, we have $I\left(r x^{*}\right)=g\left(r \mu_{x}^{*}, n\right) r Z$. Hence, the condition $I\left(r x^{*}\right)=r I\left(x^{*}\right)$ is equivalent to the condition $g\left(r \mu_{x}^{*}, n\right)=g\left(\mu_{x}^{*}, n\right)$ Because this must hold for any $0 \leq \mu_{x}^{*} \leq 1$ and any $0 \leq r \leq 1$, this means that the value of $g$ $\left(\mu_{x}^{*}, n\right)$ must remain constant for any $0 \leq \mu_{x}^{*} \leq 1$. If $Z=0$, we have $I\left(r x^{*}\right)=0$ whatever may be the value of $r$. 
Table 1

Nature of health variables: some examples

\begin{tabular}{|l|l|c|c|}
\hline & & Unbounded & Bounded \\
\hline \multirow{4}{*}{ measurement scale } & nominal & type of illness & type of illness \\
\cline { 2 - 4 } & ordinal & ordinal utility function & self-assessed health \\
\cline { 2 - 4 } & cardinal & (body) temperature & health utility index $\backslash$ binary $0 / 1$ indicator \\
\cline { 2 - 4 } & ratio-scale & health care expenditures & body length $\backslash$ life expectancy \\
\cline { 2 - 4 } & fixed & number of illnesses & visits to the medical sector in a time period \\
\hline
\end{tabular}


Table 2

Nature of health variables and rank-dependent inequality indices

\begin{tabular}{|c|c|c|c|}
\hline & & Unbounded & Bounded \\
\hline \multirow{5}{*}{ measurement scale } & nominal & inequality measurement is meaningless & inequality measurement is meaningless \\
\hline & ordinal & $\begin{array}{l}\text { inequality measurement is meaningless, } \\
\text { unless the variable is transformed into a } \\
\text { cardinal variable (see row 'cardinal') or } \\
\text { into a ratio-scale variable (see row 'ratio- } \\
\text { scale') }\end{array}$ & $\begin{array}{l}\text { inequality measurement is meaningless, unless the variable } \\
\text { is transformed into a cardinal or ratio-scale variable (see } \\
\text { below) }\end{array}$ \\
\hline & cardinal & Modified Concentration Index ${ }^{*}$ & \multirow{3}{*}{$\begin{array}{c}\text { Subset of the class of indices defined by (6b) } \\
\text { Option a: satisfying quasi-relativity: } \\
\text { Modified Concentration Index }{ }^{*} \\
\text { Option b: satisfying the Mirror Property and } \\
\text { Option b1: Quasi-absolute, and convergent: } \\
\text { Erreygers Index* } \\
\text { Option b2: Partly inverse-relative: } \\
\text { remaining indices defined by (6b) including the Wagstaff } \\
\text { Index }\end{array}$} \\
\hline & ratio-scale & Concentration Index ${ }^{*}$ & \\
\hline & fixed & $\begin{array}{l}\text { Concentration Index }{ }^{*} \text { Generalized } \\
\text { Concentration Index }{ }^{* *}\end{array}$ & \\
\hline
\end{tabular}

Note: This table assumes that unbounded and bounded indices should satisfy the Sign Condition. All indices, except the ones applied to health variables measured with a fixed scale, also satisfy Scale Invariance.

These indices are unique if one assumes that the maximum bounds of the indices are -1 and +1 .

$* *$

The Generalized Concentration Index is unique assuming the maximum bounds are $-\mu_{h}$ and $+\mu_{h}$ 